\title{
Detecting and localizing edges composed of steps, peaks and roofs
}

\author{
Pietro Perona * and Jitendra Malik ${ }^{\dagger}$
}

\begin{abstract}
It is well known that the projection of depth or orientation discontinuities in a physical scene results in image intensity edges which are not ideal step edges but are more typically a combination of steps, peak and roof profiles. However most edge detection schemes ignore the composite nature of these edges, resulting in systematic errors in detection and localization. We address the problem of detecting and localizing these edges, while at the same time also solving the problem of false responses in smoothly shaded regions with constant gradient of the image brightness. We show that a class of nonlinear filters, known as quadratic filters, are appropriate for this task, while linear filters are not. A series of performance criteria are derived for characterizing the SNR, localization and multiple responses of these filters in a manner analogous to Canny's criteria for linear filters. A two-dimensional version of the approach is developed which has the property of being able to represent multiple edges at the same location and determine the orientation of each to any desired precision. This permits junctions to be localized without rounding. Experimental results are presented.
\end{abstract}

\section{Introduction}

The problem of detecting and localizing discontinuities in greyscale intensity images has traditionally been approached as one of finding step edges. This is true both for the classical linear filtering approaches as well as the more recent approaches based on surface reconstruction.

Unfortunately, step edges are an inadequate model for the discontinuities in the image that result from the projection of depth or orientation discontinuities in physical scene. Mutual illumination and specularities are quite common and their effects are particularly

"Dipartimento di Elettronica ed Informatica, Università di Padova, and Intl' Computer Science Institute, Berkeley. Present address: MIT 35-308, Cambridge MA 02139. Email: perona@lids.mit.edu

${ }^{1}$ EECS dept., Evans Hall, U.C.Berkeley CA94720. Email: malik@robotics.berkeley.edu
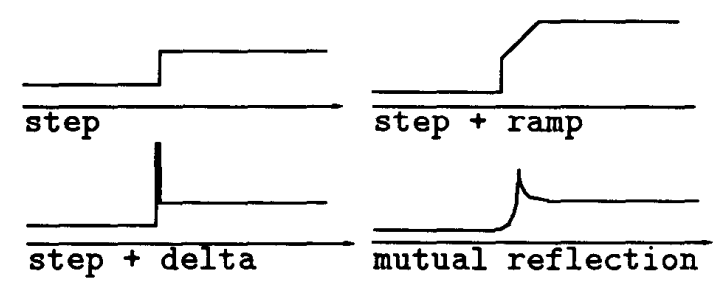

Figure 1: Some examples of edges.

significant in the neighborhood of convex or concave object edges. In addition, there will typically be a shading gradient on the image regions bordering the edge. As a consequence of these effects, real image edges are not step functions but more typically a combination of steps, peak and roof profiles (Figure 1). This had been noted experimentally by Herskovits and Binford back in 1970. Quantitative analyses of the associated physical phenomería have also been providedHorn[5] and more recently Forsyth and Zisserman [4].

The aim of this paper is to address the computational problem of detecting and localizing these composite edges.

Most local edge detection methods are based on some decision making stage following a linear filtering stage. Typically one looks for maxima in the filtered image perpendicular to the orientation of the edge. Such an approach (e.g. Canny [3] )results in a systematic error in localization whenever there is a composite edge( [11](page 9), or [2](Fig. 2. 1)). In section 2 , we prove that this problem is not specific to the Gaussian derivative filters used by Canny, but is present whatever the linear filter used. For any such filter there is a systematic localization error for composite edges. Using any (finite) number of linear filters does not help. However, we are able to show that a quadratic filtering approach is adequate. Instead of looking for maxima in $(I * f)$ one looks for maxima in $W=\left(I * f_{1}\right)^{2}+\left(I * f_{2}\right)^{2}$, or more generally $\sum\left(I * f_{i}\right)^{2}$. A special case of this approach, when two filters which are Hilbert pairs are used, gives the energy based ap- 
proach due to Morrone, Owens and their colleagues $[9,8]$.

In section 3 , we look at the problem of false responses in the presence of smooth shading. A linear filter which does not suffer from this problem is presented. A suitable quadratic filter is designed and tested on several one-dimensional examples. It correctly localizes composite edges and does not not give false responses in areas of smooth shading.

If one is to design an 'optimal' quadratic filtering approach, one needs to formulate computable forms of design criteria, analogous to the ones used by Canny [3] for linear filtering. We do this in Section 4. We are not yet able to analytically derive the 'optimal' filterhowever one can use the criteria to compare competing choices of quadratic filters.

So far, our analysis was in 1-D. To detect edges in $2 \mathrm{D}$, we use a Gaussian window to compute the 2D extension of the filter. Rotated copies of the filter are used to (conceptually) compute $W(x, y, \theta)$. A finite representation is developed which provides an optimal approximation to $W(x, y, \theta)$. At each point, the locally dominant orientations $\theta_{i}$ which correspond to the local maxima (over $\theta$ ) are determined. Allowing for multiple orientations enables junctions to be correctly localized without any rounding. Edge points are defined as the points where the directional derivative in the direction perpendicular to a locally dominant orientation is 0 . Experimental results are presented.

\section{Dealing with composite edges}

We want to detect and localize edges which are arbitrary combinations of lines, steps and roofs. For specificity and simplicity, in this section we assume that the composite edge is $I=c_{1} \delta+c_{2} \delta^{(-1)}$, though similar considerations apply for other composite edges.

A word about notation: we will write $f^{(-1)}(x)$ for $\int_{-\infty}^{x} f(t) d t$, and $f^{(-n)}(x)=\left(f^{(-n+1)}\right)^{(-1)}(x)$. So $\delta^{(-1)}$ will be the step function and $\delta^{(-2)}$ a ramp.

First we establish a proposition which show that edge localization by looking at peaks in the responses of a fixed, finite family of linear filters leads to systematic errors.

Proposition 1 For any fixed finite family of filters $\left\{f_{1}, f_{2}, \ldots, f_{k}\right\}$, there exists an image $I=c_{1} \delta+c_{2} \delta^{(-1)}$ for which none of the filter responses have a maximum at $x=0$

Proof. Edges are declared at the maxima of the response $I * f(x)=c_{1} f(x)+c_{2} f^{(-1)}(x)$. To ensure correct localization, there should be a maximum at $x=0$ for any combination of $c_{1}, c_{2}$. For a filter $f_{i}$, its response has a maximum at $x=0$ only if $\left(I * f_{i}\right)^{\prime}(0)=$ 0 . Now $\left(I * f_{i}\right)^{\prime}=c_{1} f^{\prime}+c_{2} f$, implying that the vector $\left[\begin{array}{ll}c_{1} & c_{2}\end{array}\right]^{T}$ is orthogonal to $\left[f_{i}^{\prime}(0) f_{i}(0)\right]^{T}$. To establish the proposition, one has only to pick a composite edge for which the vector $\left[\begin{array}{ll}c_{1} & c_{2}\end{array}\right]^{T}$ is not orthogonal to any of the vectors in the fixed, finite family of the $k 2 \mathrm{D}$ vectors $\left[f_{i}^{\prime}(0) f_{i}(0)\right]^{T}, i=1, \ldots, k$.

In other words, if we had available to us the outputs of $k$ different filters with a clever strategy which would enable us to pick the 'right' filter $f_{i}$ whose response should be used to localize the edge, we would still be unable to guarantee zero localization error.

Somehow the problem seems to be that for any particular linear filter we are able to construct a composite edge for which the filter is not matched. This suggests an alternative view-construct a parametrized filter which is a linear combination of an even filter $f_{e}$ (matched to $\delta(x)$ ) and an odd filter $f_{o}$ (matched to $\left.\delta^{(-1)}\right)$ and try to 'adapt' it to the particular composite edge in the image by picking the parameter value that maximizes the filter response at each point.

Call $f_{\alpha}(x)=\cos \alpha f_{e}(x)+\sin \alpha f_{o}(x)$ the filter, $I=$ $c_{1} \delta+c_{2} \delta^{(-1)}$ the image, and $U(\alpha, x)=\left(I * f_{\alpha}\right)(x)$ the response. We want to choose $\alpha$ such that at each point $x$ the response is maximized. Define $V(x)=$ $\max _{\alpha} U(\alpha, x)$ and call $\alpha(x)$ the maximizing parameter (i.e. $V(x)=U(\alpha(x), x)$ ). Notice that $\alpha(x)$ must satisfy the equation $\frac{\partial}{\partial \alpha} U(\alpha(x), x)=0$.

We would like the 'maximal' response $V(x)$ to have a maximum in zero, corresponding to the location of the edge: $V^{\prime}(0)=\left(U_{\alpha} \alpha_{x}+U_{x}\right)(\alpha(0), 0)=0$. Since $U_{\alpha}(\alpha(x), x)=0$ then it must be $U_{x}(\alpha(0), 0)=0$. Making use of the fact that $f_{o}(0)=f_{e}^{(-1)}(0)=0$ we get the following system of equations:

$$
\begin{array}{r}
U_{x}(\alpha(0), 0)=c_{1} \sin \alpha f_{o}^{\prime}(0)+c_{2} \cos \alpha f_{e}(0)=0 \\
U_{\alpha}(\alpha(0), 0)=-c_{1} \sin \alpha f_{e}(0)+c_{2} \cos \alpha f_{o}^{(-1)}(0)=0
\end{array}
$$

The maximizing value of $\alpha, \alpha(0)$, can be obtained from Equation 2. Substituting this into Equation 1 gives the following condition:

$$
f_{e}^{2}(0)=-f_{o}^{(-1)}(0) f_{o}^{\prime}(0)
$$

If this condition is satisfied, the mixed edge $c_{1} \delta+$ $c_{2} \delta^{(-1)}$ will be localized exactly by the maximum of $V(x)$ defined above.

An alternative approach yields the same condition. Define the vector of filters $F(x)=\left[f_{e}(x), f_{o}(x)\right]^{T}$. We localize features by looking for local maxima in the norm of the (vector) response to this filter of $I$. The squared norm of the response, $|I * F|^{2}$ is

$$
W(x)=\left\{c_{1} \delta+c_{2} \delta^{(-1)} * f_{e}\right\}^{2}+\left\{c_{1} \delta+c_{2} \delta^{(-1)} * f_{o}\right\}^{2}
$$

Equating the derivative of this expression with respect to $x$ at the origin to 0 gives the condition

$$
c_{1} c_{2} f_{e}^{2}(0)-c_{1} c_{2} f_{o}^{\prime}(0) f_{o}^{(-1)}(0)=0
$$

which is the same as Equation 3

Thus, we have the possibility of getting arbitrarily precise localization of composite edges simply by looking for peaks in the response to a quadratic filter, i.e. in $\sum\left(I * f_{i}\right)^{2}$. 
This is similar in form to the approach used by Morrone, Owens et al. Morrone et al [7] by a series of psychophysical experiments demonstrated that the human visual system detects features at points of strong phase congruency-these could be edges (spectral components have 0 phase), narrow bars (spectral components have 90 phase) or points on trapezoids where ramps meet plateaus (spectral components have 45 or 135 phase). To detect points of phase congruency, Morrone and Owens [9] find maxima of a local energy function $E(x)=F^{2}(x)+H^{2}(x)$ where $F(x)$ is the result of a convolution $I * f(x)$, and $H(x)$ is its Hilbert transform (equivalently $I$ could be convolved with the Hilbert transform of $f$ ). Morrone and Owens show good empirical results for a particular choice of $f$.

While their reasoning was in the Fourier domain and aimed at detecting phase congruency based on a psychophysical definition of a feature, we arrive at a similar formulation purely motivated by a computational criterion of localizing composite edges exactly.

From our formulation it follows that there is nothing particularly sacred about the use of Hilbert filter pairs as done by Morrone, Owens et al. In fact, if the composite edge consists of, say a bar and a step edge at quite different scales, one should probably use $f_{e}$ and $f_{o}$ tuned to different widths (scales) and thus not Hilbert pairs. To make a proper choice of these filters, one should instead bring to bear the criteria of having a good signal-to-noise ratio, low stochastic localization error etc. analogous to the approach used by Canny for linear filters.

\section{Dealing with shading gradients}

A well known problem of first derivative edge detectors is that they respond with false edges in areas with smooth shading even when the gradient of brightness is constant. To avoid these false positives, one may have to set a threshold which leads to the rejection of genuine low-contrast edges. This problem has persisted in the 'modern' approaches based on surface reconstruction. Whether the formulation is a probabilistic one using MRFs (e.g. Geman and Geman) or a variational one (e.g. Blake and Zissserman [2]), if the cost function includes terms like the squared gradient there will be a tendency towards piecewise constant reconstructions.

In the linear filtering framework, Binford [1] describing the Binford-Horn line finder discusses one solution to this problem- a lateral inhibition stage preceding the stage of finding directional derivatives. Essentially this amounts to using third derivatives, and suffers from the expected weakness--low signal to noise ratio compared to first derivative operators. A simple calculation using the SNR criterion defined by Canny [3] confirms this.

A compact characterization of filters which do not suffer from the linear gradient problem can be obtained as follows: suppose that the image just consists of a
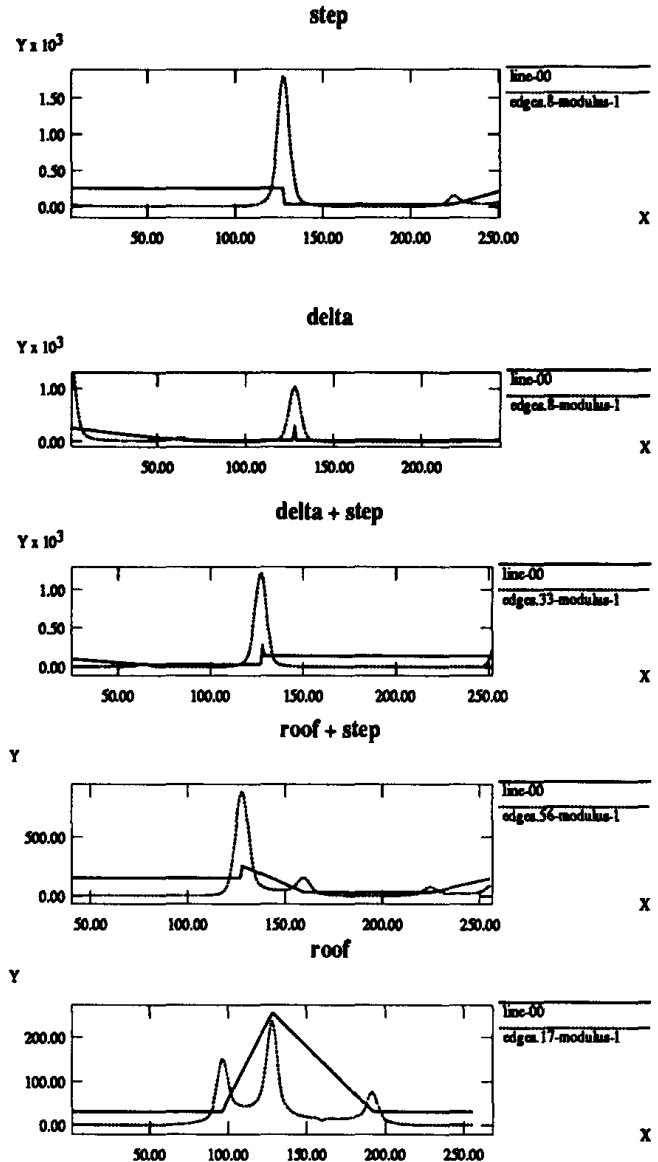

Figure 2: 1 dimensional examples. The energy peaks correspond to the edge position and the constant gradient areas generate zero energy.

ramp function $I(x)=\delta^{(-2)}(x)$. The response of a linear filter $f$ to such a ramp is $I * f=f^{(-2)}(x)$. It can be seen that $f^{(-2)}(x)$ should satisfy the following two conditions:

1. $\left\|f^{(-2)}(x)\right\| \rightarrow 0$ for $\|x\| \rightarrow \infty$. This ensures that far enough from the roof junction, the response to a ramp is negligible.

2. $f^{(-2)}(x)$ either has a zero crossing or a maximum or a minimum at the origin. This is to enable the localization of onset of the ramp without any bias.

While the third derivative of a Gaussian $G_{\sigma}^{\prime \prime \prime}(x)$ is one filter which would satisfy these criteria, there are others which do so without that significant a drop in SNR. One such choice is the Hilbert Transform of $G_{\sigma}^{\prime \prime}(x)$ which is an odd-symmetric filter. We computed Canny's SNR and localization criteria for this filter and compared it with $G_{\sigma}^{\prime}(x)$. It turns out that for $G_{\sigma}^{\prime}(x)$, the SNR is $1.062 \sigma^{0.5}$ and local- 
ization is $0.8673 \sigma^{-0.5}$. For $\left(G_{\sigma}^{\prime \prime}\right)_{H}(x)$, the SNR is $0.6920 \sigma^{0.5}$ and localization is $0.87535 \sigma^{-0.5}$. Considering the product of the SNR and the localization, the numbers are 0.92 and 0.606 respectively implying that $\left(G_{\sigma}^{\prime \prime}\right)_{H}$ is worse by about $34 \%$. However, its $r$ value is 0.676 which is $32 \%$ better than $r=0.51$ for the $G_{\sigma}^{\prime}$. In other words, while the $\left(G_{\sigma}^{\prime \prime}\right)_{H}$ is roughly comparable to the $G_{\sigma}^{\prime}$ filter used by Canny, its immunity to smooth shading makes it preferable.

For a particular choice of quadratic filter, namely $f_{e}=G_{\sigma}^{\prime \prime}$ and $f_{o}=\left(G_{\sigma}^{\prime \prime}\right)_{H}$, Figure 2 shows the response to a number of different stimuli. Note how in each case, the composite edge is correctly localized and that the filter is insensitive to linear shading.

\section{Computation of the performance criteria}

In the choice of a filter one would like to minimize different types of edge-detection errors. What follows is a list of criteria for evaluating quadratic filteringbased edge-detectors

Signal to noise ratio - Ratio of signal response to the variance of the response due to noise.

Stochastic Localization error - Localization error due to noise.

Systematic Localization error - Error committed in locating the edge in the no-noise situation.

Multiple responses - Edges detected in the neighbourhood of a true one due to noise in the data.

After establishing some notation we report the formulae for computing the criteria for a given filter. For a derivation of the formulae see [6].

\subsection{Notation}

Edge $-G(x)=c_{1} \delta^{(-2)}(x)+c_{2} \delta^{(-1)}(x)+c_{3} \delta(x)$

Noise $-N(x)=n_{0} \eta(x), \eta(x)$ being white zero-mean unit-variance Gaussian noise.

Image $-I(x)=G(x)+N(x)-$ Signal + noise.

Filters $-\mathbf{f}(x)^{T}=\left[f_{1}(x), \ldots, f_{n}(x)\right]$, and, for convenience, $\mathbf{F}(x)^{T}=\left[F(x)_{1}, \ldots, F(x)_{n}\right]$, with $\mathbf{F}^{\prime \prime}(x)=\mathbf{f}(x)$

Responses $-\mathbf{r}_{G}(x)=(\mathbf{f} * G)(x), \mathbf{r}_{N}(x)=(\mathbf{f} * N)(x)$, $\mathbf{r}(x)=\mathbf{r}_{G}(x)+\mathbf{r}_{N}(x)$

Power $-W(x)=\|\mathbf{r}(x)\|^{2}$

Correlations - The $n x n$ correlation matrix $\mathbf{R}(t)$ defined componentwise by:

$$
\mathbf{R}_{i j}(t) \doteq\left\langle f_{i}(\cdot+t), f_{j}(\cdot)\right\rangle_{L_{2}}
$$

$\mathbf{R}^{\prime \prime}(t)$ defined similarly as:

$$
\mathbf{R}^{\prime \prime}{ }_{i j}(t) \doteq\left\langle f_{i}^{\prime}(\cdot+t), f_{j}^{\prime}(\cdot)\right\rangle_{L_{2}}
$$

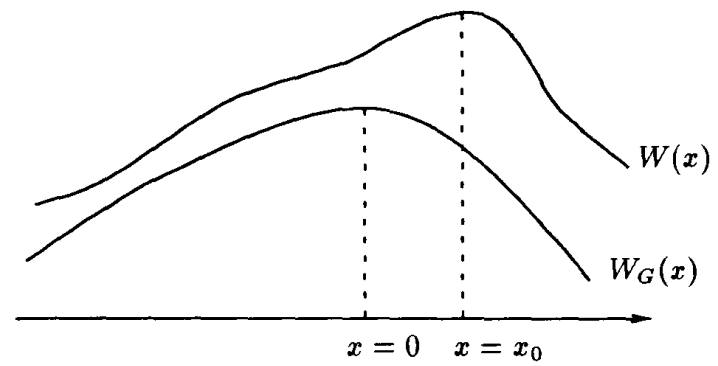

Figure 3: Localization error due to noise.

\subsection{Signal to noise ratio}

Define signal to noise ratio as the ratio of the response to pure signal at the edge and the standard deviation of the response to pure noise. In the special case that the edge is a combination of roof, step and line: $G=$ $c_{1} \delta^{(-2)}+c_{2} \delta^{(-1)}+c_{3} \delta$ the signal to noise ratio is:

$$
S N R=\frac{\|\mathbf{H}(0) \mathbf{c}\|}{n_{0} \sqrt{\operatorname{tr}(\mathbf{R}(0))}}
$$

Where $\mathbf{c}$ indicates the vector of components $c_{1}, c_{2}, c_{3}$, and $\mathbf{H}$ is defined componentwise by $\mathbf{H}_{i j}=$ $F_{j}^{(i-1)}, i=1, \ldots, 3$ and $j=1, \ldots, n$.

\subsection{Stochastic localization error}

Label $x=0$ the position where the response $W_{G}(x)$ to noiseless signal peaks (i.e. $W_{G}^{\prime}(0)=0$ ), and $x=$ $x_{0}$ the coordinate where the response to noisy signal $W(x)$ does (i.e. $W^{\prime}\left(x_{0}\right)=0$, see Fig 3 ).

The expectation of the stochastic localization error is zero, and the variance is:

$$
E x_{0}^{2} \approx n_{0}^{2} \frac{E\left(\left(h_{x_{0}}^{\prime} * \eta\right)\left(x_{0}\right)\right)^{2}}{W_{G}^{\prime \prime}(0)^{2}}=n_{0}^{2} \frac{\mathbf{R}_{h_{0}}^{\prime \prime}(0)}{W_{G}^{\prime \prime}(0)^{2}}
$$

where $h_{x_{0}}$ is defined by:

$$
\left(h_{x_{0}} * \eta\right)\left(x_{0}\right) \doteq 2(G * \mathbf{f})^{T}\left(x_{0}\right)(\mathbf{f} * \eta)\left(x_{0}\right)
$$

$\mathbf{R}_{h_{0}}^{\prime \prime}(0)$ is the autocorrelation matrix of $h_{x_{0}}^{\prime}$.

\subsection{Systematic localization error}

Consider a signal $G$ defined as in section 4.1 ; whatever the choice of the coefficients $c$, the edge is located at $x=0$. Call $x_{e}$ the position of the corresponding maximum of $W(x)$, which we use to localize the edge; $x_{e}-0=x_{e}$ is a systematic localization error.

A necessary and sufficient condition for $x_{e}$ to be a maximum point is that $W^{\prime}\left(x_{e}\right)=0$ and $W^{\prime \prime}\left(x_{e}\right)<$ 0 . Expanding $W^{\prime}$ in Taylor sum around $x=0$ and computing it in $x=x_{e}$ we obtain:

$$
0=W^{\prime}\left(x_{e}\right)=W^{\prime}(0)+W^{\prime \prime}(0) x_{e}+\mathcal{O}\left(x_{e}^{2}\right)
$$


which gives us an estimate of $x_{e}$ in terms of the derivatives of $W$ at the origin:

$$
x_{e} \approx \frac{W^{\prime}(0)}{-W^{\prime \prime}(0)}=-\frac{\mathbf{c}^{T} \mathbf{H}^{\prime T}(0) \mathbf{H}(0) \mathbf{c}}{\mathbf{c}^{T} \mathbf{H}^{\prime T}(0) \mathbf{H}(0) \mathbf{c}+\left\|\mathbf{H}^{\prime}(0) \mathbf{c}\right\|^{2}}
$$

where $\mathbf{c}^{\boldsymbol{T}}=\left[c_{1}, c_{2}, c_{3}\right]$ and $\mathbf{H}$ is defined componentwise by $\mathbf{H}_{i j}=F_{i}^{(j-1)}, j=1, \ldots, 3$ and $i=1, \ldots, n$.

A sufficient condition for the systematic localization error to be zero is therefore (see [6]) that the filter collection $f$ satisfies the conditions:

$$
\sum_{k=1}^{n} F_{k}^{i}(0) F_{k}^{(j-1)}(0)=-\sum_{k=1}^{n} F_{k}^{j}(0) F_{k}^{(i-1)}(0) \quad i, j=1 \ldots 3
$$

This is a more general form of equation 3 .

\subsection{Spacing of the maxima in the neighbourhood of an edge}

We suppose that the noise variance, $n_{0}$, is small with respect to the magnitude of the signal. Therefore we approximate the value of $W(x)$ disregarding the terms that are quadratic in $n_{0}$ :

$$
\begin{aligned}
W(x) & \approx\|(G * \mathbf{f})\|^{2}+2 n_{0}(G * \mathbf{f})^{T}(\mathbf{f} * \eta)= \\
& =W_{G}(x)+2 n_{0}\left[G * \mathbf{f}^{T} \mathbf{f}\right] * \eta= \\
& =W_{G}(x)+n_{0} h_{x} * \eta
\end{aligned}
$$

Where $h$ is the scalar space-varying kernel defined as $h_{x}(\tau) \doteq 2(G * \mathbf{f})^{T}(x) \mathbf{f}(\tau)$.

We may apply Rice's formula to compute the expected value of the distance between maxima of the random process $W_{\Delta}(x)=W(x)-W_{G}(x)=n_{0} h_{x} * \eta$ :

$$
d_{W_{\Delta}}(x)=2 \pi \sqrt{\frac{R_{h_{x}}(0)}{R_{h_{x}}^{\prime \prime}(0)}}
$$

The expectation has an argument $x$ since it depends on the distance from the location of the edge $G(x)$. In a neighbourhood of the edge we expect the derivative of $W_{G}$ to be close to zero and thus the estimate of the spacing of the maxima of $W_{\Delta}(x)$ to be a good estimate of the spacing of the maxima of $W(x)$.

Define $\vec{\alpha}=G * \mathbf{g}$, then $h=2 \vec{\alpha}^{T} \mathbf{f}$. The autocorrelations are:

$$
\begin{gathered}
\frac{1}{4} R_{h_{x}}(0)=\vec{\alpha}^{T} \mathbf{R}_{f}(0) \vec{\alpha} \\
\frac{1}{4} R_{h_{x}}^{\prime \prime}(0)=\vec{\alpha}^{T} \mathbf{R}_{f}(0) \vec{\alpha}^{\prime}+\vec{\alpha}^{\prime T} \mathbf{R}_{f}^{\prime}(0) \vec{\alpha}+\vec{\alpha}^{T} \mathbf{R}_{f}^{\prime \prime}(0) \vec{\alpha}
\end{gathered}
$$

\section{Detecting edges in two dimensions}

To detect edges in 2D, we use a Gaussian window to compute the 2D extension of the filter $F(x, y)=$ $f(x) G_{\sigma_{2}}(y)$. We use two kernels, even and odd; rotated copies $F_{\theta}^{e}, F_{\theta}^{o}$, of the filter are used to compute the square modulus $W(x, y, \theta)=\left(F_{\theta}^{e} * I\right)^{2}+\left(F_{\theta}^{o} * I\right)^{2}$, where $I$ is the image. In practice one cannot afford to compute convolutions of the image with filters at an infinity of orientations. It turns out that it is possible to approximate kernels $F(x, y, \theta)$ with arbitrary precision using linear combinations of a finite number of functions. This technique is based on the singular value decomposition of the linear operator associated to the kernel and is described in detail in [10]. What is important to remark here is that it is possible to reason on a continuum of orientations.

\subsection{Edge detection}

At edge points the filter output 'energy' $W$ will have a maximum at the orientation $\theta_{e}$ parallel to the edge. Fix $\theta_{e}$ and consider $W\left(x, y, \theta_{e}\right)$. Along a line orthogonal to the edge the problem reduces to the $1 \mathrm{D}$ case: there will be an energy maximum at the edge. Edges can be found by marking as 'edge points' all the points $p=(x, y, \theta)$ that satisfy:

$$
\frac{\partial}{\partial \theta} W(p)=0, \quad \frac{\partial}{\partial \mathbf{v}_{\theta}} W(p)=0
$$

where $\mathbf{v}_{\theta}$ is the unit vector orthogonal to the orientation associated to $\theta$.

The search for the edge points has been implemented as follows:

1. For each image pixel $(x, y)$ the angles $\theta_{i}(x, y)$ at which the response is maximized are found. For this operation we use Brent's method which cleverly combines golden section search with inverse parabolic interpolation. The upper bound on the orientation error was set at 1 degree. The angle space is coarsely sampled (approx. a sample every 5 degrees) to provide initial conditions for the bracketing algorithm. The energies $W_{i}(x, y)$ corresponding to $\theta_{i}(x, y)$ are also stored. The lower $70 \%$ of the sampled energies at each point are averaged to give a global noise estimate.

2. Points $\left(x, y, \theta_{i}(x, y)\right)$ are marked as edge points only if the associated energy is greater than the energy at the two neighbouring pixels in the direction orthogonal to $\theta_{i}$.

3. The edge pixels are thresholded.

4. The position of the edges is refined to subpixel accuracy by fitting a parabolic cylinder to $W\left(x, y, \theta_{i}\right)$ at the edge pixels. The axis of the cylinder is taken to be the local estimate of the edge position. In our implementation $3 \times 3$ neighbourhoods were used and the three parameters of the parabola estimated; see [6] for the details.

We have tested the algorithm on both natural and synthetic data. We used a quadratic filter with $f_{e}=$ $G_{\sigma_{1}}^{\prime \prime}, f_{o}=\left(G_{\sigma_{1}}^{\prime \prime}\right)_{H}$, and the 2D extension computed using a windowing function $G_{\sigma_{2}}$ with $\sigma_{2}: \sigma_{1}=2$ or 3 . One of our series of experiments used a synthetic image of three concentric circles (alternate rings black 
and white) with added Gaussian noise. Comparison of estimates of orientation and curvature from our algorithm with the ground truth yielded very good results [6]. For example, for a SNR of 6.4, and using a quadratic filter $\left(\sigma_{1}=2, \sigma_{2}=6\right.$ pixels) orientation could be estimated on circles of radii 60 and 90 pixels with median absolute error of $0.8^{\circ}$ and on a circle of radius 30 pixels with an error of $1.1^{\circ}$. Curvature, which was estimated pointwise by a finite difference approximation using orientation estimates in a $2 \times 2$ window could be measured to a median error of $22 \%$, $18 \%$, and $14 \%$ respectively for the circles of radii 90 , 60 and 30 pixels.

In Figure 5 we compare the edges obtained by our edge detector with those found by the Canny edgedetector. While the false positives are largely a matter of threshold selection (in this case), note how the junction is broken up and rounded by the Canny edge detector. Figure 4 shows a comparison for a more complex image.

\section{Acknowledgements}

The experimentation reported in this paper was carried out using Paul Kube's 'viz' image processing package. This research was partially funded by an NSF-PYI grant.

\section{References}

[1] T. Binford. Inferring surfaces from images. Artificial Intelligence, 17:205-244, 1981.

[2] A. Blake and A. Zisserman. Visual reconstruction. MIT press, 1987.

[3] J. Canny. A computational approach to edge detection. IEEE trans. PAMI, 8:679-698, 1986.

[4] D. Forsyth and A. Zisserman. Mutual illumination. In Proceedings of the IEEE CVPR, pages 466-473, 1989.

[5] B. Horn. Image intensity understanding. Artificial intelligence, 8(2):201-231, 1977.

[6] J. Malik and P. Perona. Detecting and localizing edges composed of steps, peaks and roofs. Technical Report UCB/CSD 90/590, Computer Science Division (EECS), U.C.Berkeley, 1990.

[7] M.C.Morrone, D. Burr, J. Ross, and R. Owens. Mach bands are phase dependent. Nature, (324):250-253, 1986.

[8] M. Morrone and D. Burr. Feature detection in human vision: a phase dependent energy model. Proc. $R$. Soc. Lond. B, pages $221-245,1988$.

[9] M. Morrone and R. Owens. Feature detection from local energy. Pattern Recognition Letters, 6:303-313, 1987.

[10] P. Perona. Finite representation of deformable functions. Technical Report 90-034, International Computer Science Institute, 1947 Center st., Berkeley CA 94704, 1990.

[11] J. Ponce and M. Brady. Towards a surface primal sketch. Technical Report 824, MIT Artificial Intelligence Laboratory, 1985.



(a)

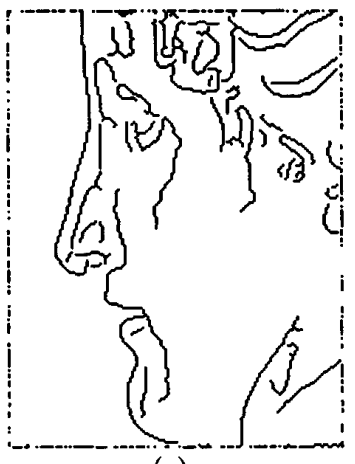

(c)

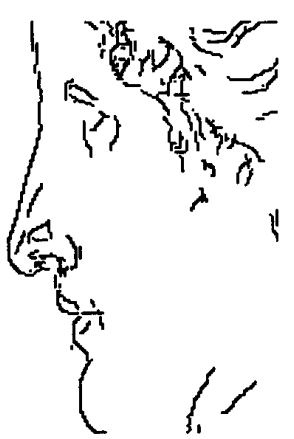

(b)
Figure 4: Comparison of the Canny detector and our $2 \mathrm{D}$ detector. (a) Original (Paolina Borghese, Canova circa 1800). (b) our detector, $\sigma_{1}=1, \sigma_{2}: \sigma_{1}$ ratio $2: 1$. (c-d) Canny detector with $\sigma=1$, and threshold $(150,250)$, and $(200,400)$ respectively.

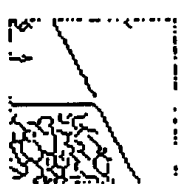

(1)



(1)



(3)

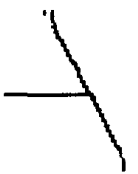

(3)



(5)

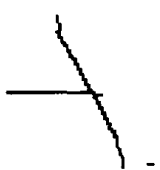

(5)
Figure 5: T-junction example. Top: Canny detector with $\sigma=1,3,5$. Bottom: our $2 \mathrm{D}$ detector, same $\sigma_{1}$, $\sigma_{2}: \sigma_{1}$ ratio $3: 1$ 